\title{
Auditing Quality of Research in Social Sciences
}

\author{
SANNE AKKERMAN ${ }^{1, \star}$, WILFRIED ADMIRAAL ${ }^{2}$, \\ MIEKE BREKELMANS ${ }^{1}$ and HEINZE OOST ${ }^{1}$ \\ ${ }^{1}$ IVLOS Institute of Education, Utrecht University, Po Box 80127, 3508 TC Utrecht, The \\ Netherlands; \\ ${ }^{2}$ Graduate School for Teaching and Learning, Unversity of Amsterdam, Wibautstraat 2-4, \\ 1091 GM, Amsterdam
}

\begin{abstract}
A growing body of studies involves complex research processes facing many interpretations and iterations during the analyses. Complex research generally has an explorative in-depth qualitative nature. Because these studies rely less on standardized procedures of data gathering and analysis, it is often not clear how quality was insured or assured. However, one can not easily find techniques that are suitable for such complex research processes to assess the quality of the study. In this paper, we discuss and present a suitable validation procedure. We first discuss how 'diagnosing' quality involves three generic criteria. Next, we present findings of previous research in possible procedures to assure the quality of research in social sciences. We introduce the audit procedure designed by Halpern [(1983) Auditing Naturalistic Inquiries: The Development and Application of a Model. Unpublished doctoral dissertation, Indiana University] we found an appropriate starting point for a suitable procedure for quality judgment. Subsequently, we will present a redesign of the original procedure, with according guidelines for the researcher (the auditee) and for the evaluator of the quality of the study (the auditor). With that design, we aim to enable researchers to bring forward their explorative qualitative studies as stronger and more equally valuable to studies that can rely on standardized procedures.
\end{abstract}

Key words: Audit, quality procedure

\section{Introduction}

Qualitative and in-depth analyses are increasingly used in social sciences. However, in a large number of studies the question how quality was insured or codified is not answered (see also Sale and Brazil, 2004). Specifically in studies that rely on intensive data gathering and in-depth qualitative analysis, the development of manageable units of analysis and categories often requires a lot of interpretations and iterations during the

*Author for correspondence: Sanne Akkerman, IVLOS Institute of Education, Utrecht University, Po Box 80127, 3508 TC Utrecht, The Netherlands; E-mail: s.f.akkerman@ ivlos.uu.nl 
process of analysis. Consequently, this type of research is more subject to decisions that undermine trustworthy findings compared to research where more standardized methods of analyses are used. If validation procedures are not part of the research, it is difficult to judge the quality of a study, and researchers themselves are not triggered to maintain the quality of their studies. Therefore, procedures to assess quality may be of help. Being able to use a quality procedure will enable researchers to bring forward in-depth and qualitative studies stronger and more equally valuable to studies that can rely on generally accepted (often quantitative) procedures. Despite the apparent need within qualitative and in-depth research for procedures to determine quality, it is difficult to find appropriate validation techniques within literature databases for social sciences. Therefore, we concluded that we need to develop a procedure ourselves. We will introduce a so-called audit procedure that we found to be a starting point for an appropriate procedure for quality judgment. Following, we will present a more spelled out version of this procedure, with according guidelines for the two sides involved in the evaluation of the quality of the study. Before introducing the audit procedure, the paper first addresses criteria for quality and the object of quality assessment.

\section{Criteria for Quality}

In order to determine the quality of scientific research three generic criteria have to apply to research decisions: visibility, comprehensibility, and acceptability. Although determining quality is a requirement for all research, it becomes a specific matter of attention when the process of analysis involves many interpretations and iterations, where one can not rely on standardized strategies and procedures. Consequently, the process of analysis becomes less transparent and the quality maintenance has not been built in during the process. Hence, it is more difficult to assure the quality of the research process and its results.

These kind of complex research processes can be evoked when a research object concerns a complex problem or in case of explorative studies, in which the research question is initially formulated in broad terms and where it is not clear beforehand which type of answer it will produce. One can also face complex processes in studies where there are several types of data to be related in the analyses in order to answer the research question.

In line with Funke (1991), we characterize complex research processes as processes with intransparancy, polytely, situational complexity and effect delay. Intransparancy occurs in situations where only information on symptoms is available, in situations where but a few (of the) variables lend themselves for direct observation, or in situations where a huge amount 


\section{AUDITING QUALITY}

of variables forces the researcher to select a limited amount of relevant variables. One speaks of polytely, in case multiple goals are presented that (may) interfere with each other. Situational complexity is defined by the (complicated) interrelations between variables. And one speaks of effect delay when not every act shows direct consequences. Middleton (2002) explained that the complexity exists "precisely because it is difficult to define each of the elements of the problem space." The consequence of complex research processes is that one is supposed to first penetrate the problem and take into account the possibility that the problem can be conceptualized in more than one way, and that in most cases a "single, correct answer does not exist." Besides, when faced with complex research processes, existing solution strategies may not be adequate and new concepts, tools and strategies in the process of data gathering, and data analysis will have to be designed and constructed.

Application of the quality criteria that are in our perspective most basic for scientific research requires complex research processes to meet criteria regarding visibility, comprehensibility and acceptability of research decisions. Acceptability entails that the decisions are substantiated by the researcher according to the standards, norms and values in the particular field of study. Generally, this implies that substantiation for a research decision follows the argumentative logic of the field and, in its content connects to what is considered as known and not known in the research domain. Pre-conditional criteria directly derived from the acceptability criterion are visibility and comprehensibility. A decision must be made explicit and communicated to be judged at all and substantiated to be judged by its logic and content.

In research that can rely on standardized procedures for data gathering and data analysis, both pre-conditional criteria often are not relevant, since the standardized nature of the procedures encompasses explicit and substantiated steps. With complex research processes there are no standardized procedures to rely on. It is therefore more difficult for the researcher to know how quality was maintained or may have been damaged during the research process. Both the visibility of the decisions taken, as well as their substantiation may easily be problematic. In this kind of research, the researcher is a crucial "instrument" in the creation of conceptual and strategic solutions. This makes it tricky for the researcher to simultaneously be reflective and aware of the quality insurance. Also, conducting a systematic quality check afterwards is not easy to do, since the process is more opaque.

The complex nature of these research processes generates a complicated quality check. It is not possible to repeat the analysis using a certain procedure or technique that the researcher claims to have followed, in order to see whether all conditions were met and all steps have been successfully gone through. When it is a standard part of a research process to articulate the 
decisions taken and describe the underlying principles, it not only allows evaluating the quality of the study, but also enables the researcher to consciously consider how to maintain and improve the quality.

Before going into procedures that allow for quality maintenance as well as quality judgment in terms of visibility, comprehensibility, and acceptability, we will first specify different objects of quality assessment depending on different phases of the research process.

\section{Objects of Quality Assessment}

Considering an instrument or procedure for quality evaluation of research requires specification of the object of quality assessment. The object of quality assessment is different for different phases in the research process. Generally, a research process involves:

- designing and writing the research proposal;

- gathering data;

- analyzing data up to deriving results and conclusions, and

- reporting the research

Determining the quality of the research proposal is often done either by supervisors, by committees of research institutes or by organizations that offer funding for carrying out the research proposal. To enhance the chance for a positive judgment, proposals are often discussed with peers and supervisors in the role of "critical friends."

The report of the research is generally accessible for the complete scientific forum. It is conventional that preceding the publication, a quality check of the report took place within the inner circle of researchers (colleagues, supervisors), or externally by means of committees (in case of a doctoral thesis), editorial boards of journals (in case of articles), publishers (in case of books), etcetera.

In the phase of data gathering and analysis, however, there are several decisions and (in between) products that remain invisible for at least the scientific forum, but often also for colleagues and supervisors, who are in some way involved in the research project. In case the analysis concerns quantitative data, many procedures have already built-in quality checks. In case of qualitative in-depth studies this is frequently not the case. Determining interrater-reliability, for example, is not always an option, simply because of the interpretative and iterative nature of the data analysis. The activities with critical friends are often unstructured and difficult to describe as a quality "procedure" in the report of the research. Because of the absence of suitable procedures in case of qualitative data, we think it is necessary to design a procedure for a quality check for the decisions taken during the data gathering and analyses. In its broadest sense, the procedure 


\section{AUDITING QUALITY}

should be useful for evaluating quality of the whole process of data gathering and analysis. Thereby, the departing question for evaluation is whether the results and conclusions that the scientific forum can read in the final product are grounded in acceptable decisions during the data gathering and data analysis. In other words, the linkages between the visible outcomes of the study and the invisible decisions from which they were derived are object of evaluation.

\section{Development of a Procedure}

Searching for quality procedures in social scientific literature ${ }^{1}$ revealed but one worked-out procedure relevant for maintaining and evaluating quality of complex research. The one procedure we found, the so-called audit procedure, stems from a doctoral dissertation from Halpern dating back to 1983. According to Halpern (1983), Lincoln and Guba (1985), Schwandt and Halpern (1988), and Miller (1997) conducting an audit is the major technique to ascertain if studies meet the criterion of trustworthiness. We found this procedure initially to be the most developed and useful tool for maintaining and evaluating the quality of research that involves complex analyses. Searching for similar notions as the audit, specifically peer debriefing and reflective journals, though being referred to as useful means for enhancing quality, ${ }^{2}$ did not yield elaborate alternatives to the audit.

The audit was originally developed by Halpern as a response to quality issues arising from a growing interest in conducting naturalistic research (ethnographic, phenomenological and anthropological studies). The notion of an audit was built on the metaphor of a fiscal audit (Guba, 1981; Lincoln and Guba, 1985). In a fiscal audit, an auditor is called in to validate the accounts of a business or industry to perform two tasks. A first task is to examine the method of accounting or the process by which the accounts were kept to verify if the business followed fair and generally accepted practices. A second task is to examine the products of accounting to verify that they are supported by corroborative documents. These two tasks can be translated to an audit judging respectively the reliability and objectivity criterion for social scientific research.

Subsequently, Halpern introduced the confirmability (objectivity) audit as (p. 12): "an audit certifying that data exist in support of every interpretation and that the interpretations have been made in ways consistent with the available data. This type of audit is concerned primarily with the products of the inquiry, and requires extensive documentation (Guba, 1981)." Similarly, Halpern introduced the dependability (reliability) audit (p.15): "to examine the processes whereby data were collected and analyzed and interpretations were made" and "....to examine the audit trail and to 
comment upon the degree to which procedures used fall within "generally acceptable" practice. Such a reliability audit deals primarily with the processes of the inquiry (Guba, 1981)."

Based on literature from naturalistic methods, literature about auditing, literature about managing collaborative relationships and records analysis, Halpern developed an audit procedure. This procedure includes both an audit for objectivity and reliability as a set of evaluation questions concerning measures taken by the researcher (auditee) for maintaining truth value (in common terms "internal validity" and in naturalistic terms "credibility"). He developed this procedure by applying it in three different studies. This resulted in the audit technique that is presented and extensively described by Halpern (1983), Lincoln and Guba (1985), and Schwandt and Halpern (1988).

\subsection{AUDIT PROCEDURE}

Generally, the audit procedure concerns a trajectory in which an auditee, the researcher whose research is object for quality assessment, contacts an auditor, who will perform the audit. For the auditor to be able to perform the audit, the auditee needs to prepare beforehand a so-called audit trail, in which the whole procedure of data gathering and analysis is documented, including both raw data material, categorized data material, and the findings. The procedure starts with an initial orientation to the study and negotiation with the auditee about the aim of the audit and the procedure to be followed. Here the auditee and the auditor agree upon goals, roles, and rules of the audit. During the orientation stage, the auditor has to become familiar with the study, by receiving all the audit trail components along with an explanation of the recordkeeping system. Following, the auditor will have to look in detail in all the materials provided in the audit trail, determining its "auditability." This determination entails deciding whether the audit trail is complete, and understandable, entailing it is structured according to clear systematic interrelations among the components. Determining the auditability may lead to additional explanation by the researcher about the trail or to some revisions of the structure of the audit trail. The next stage is the contract negotiation which is a more or less formal agreement on the timeline of the audit, the aims of the audit procedure, the roles of both auditee and auditor, the format of the audit report and the criteria for renegotiation. Then in the major stage, the auditor assesses the trustworthiness of the study in terms of the specific quality criteria. Finally, the auditor writes an auditor report and discusses this with the auditee. This may lead to a renegotiation (and possibly to a new audit procedure). The final auditor report contains a substantiated assess- 
AUDITING QUALITY

Table I. Stages of the audit procedure

\begin{tabular}{ll}
\hline Stage & Description \\
\hline 1. Orientation to the audit procedure & Both auditee and auditor negotiate \\
& and agree upon goals, roles and rules \\
& of the audit. \\
& Auditee arranges the logistics for the \\
2. Orientation to the study & auditor and explains the audit trail, \\
& and auditor becomes familiar with the \\
& study. \\
3. Determination of the auditability of & Auditor determines the complete- \\
the study & ness, comprehensibility and utility of \\
& the audit trail. Auditee and auditor \\
& discuss the auditability. \\
& Auditee and auditor establish timeline, \\
4etermine goals, specify roles, arrange & logistics, determine outcomes and \\
& format, and identify renegotiation \\
& criteria. \\
& Based on the audit trail auditor \\
assesses the research process in terms \\
of the specific quality criteria. \\
Auditor presents findings and dis- \\
cusses discrepancies; Auditee assesses \\
the accuracy of the auditor claims \\
and adherence to the agreement. This \\
conversation might result in rede- \\
signing the research process (leading \\
to another audit), adjustment of the \\
auditor report, or a modification of \\
the agreement. \\
Auditor writes a substantiated assess- \\
ment on the trustworthiness of the \\
study.
\end{tabular}

ment. When part of the agreement in the contract, the auditor report may also include feedback about how to adjust the research process in case it is evaluated having too little quality. This audit trajectory can be considered as a process including seven different stages. The stages are summarized in Table I. Stage 5, the assessment, forms the central part of the audit procedure. 


\subsection{LiteratURE ON THE AUDIT PROCEDURE}

Besides the publications in the early 1980s, we found but two more recent explicit discussions of the audit as a procedure. The first one was provided by Rodgers and Cowles (1993), who explore and discuss both the types of documentation a comprehensive audit trail for a qualitative study generally includes, and some basic strategies for developing an audit trail. The basic types of documentation are contextual, methodological, analytic, and personal response documentation. Recommended documentation strategies are compulsive recording, use of a comfortable but consistent recording system, detailed description, immediate recording prior tot digestion or discussion, and ongoing cross-indexing between notes and data.

The second explicit discussion of the audit as a procedure was provided by Miller (1997), who stated that the concept of using the audit to assess the trustworthiness of an inquiry has not been operationalized as a verification strategy extensively, despite its introduction dating back to 1983 . Based on the original framework offered by Halpern (1983), Lincoln and Guba (1985), and Schwandt and Halpern (1988), she audited 11 dissertations. She presents a reflection on the procedure, based on her own experiences as auditor, interviews with eight auditees and with three faculty members recommending the audit to students. Although offering some additional suggestions to the original audit procedure, her account of the audit points mostly to its value:

The external audit is one of the most systematic, rigorous verification strategies, and places specific demands on the researcher to keep good records, and establish a clear audit trail. It challenges the researcher to document the research process, and be intentional and careful about record keeping. It keeps them honest and accountable. However, it does much more. It provides support, validation and feedback, particularly through a lonely process. It gives researchers' confidence in their work and abilities, and confidence going into the oral defense. It helps faculty who are uncomfortable or unfamiliar with qualitative research address some of their concerns about the validity of the process and product. It provides readers with a lens from which to view the credibility of the account. (Miller, 1997, p. 15)

\subsection{REDESIGN OF THE AUDIT PROCEDURE}

To see whether the procedure can be considered appropriate for quality maintenance and evaluation in complex research, we revisited the details of the procedure as originally presented by Halpern (1983). We found that the procedure was not yet fully specified in several aspects. For one, in the description of Halpern it is unclear what the precise object is for evalua- 
tion in the audit procedure. Second, no clear distinction was made between data gathering and analysis in the design of the audit procedure and the audit trail. This makes it unclear which components within the process of data gathering and analysis have to be evaluated. Moreover, this issue was not resolved in the audit procedure as presented by Miller (1997) or by the suggestions of Rodgers and Cowles (1993). So, we decided to further specify the audit procedure. We also decided to describe it in a way that it is more broadly applicable. Although the original procedure was designed for naturalistic research, we believe it is suitable for all kinds of complex research.

As test cases for applying, evaluating, and refining the audit procedure, we used two studies in which a complex, in-depth qualitative analysis was conducted. Both studies focused on collaboration projects of academics (Akkerman, 2006; Akkerman, Admiraal, Simons, \& Niessen, in press) and faced a lot of different data like individual interviews, email communication, project documentation, observations and video tapes of project meetings. The data gathering process used an ethnographic approach, with the researcher as an observant of the collaboration for 2 years. The analyses concerned several steps to process and interrelate the different types of data in order to construct a coherent story about the project group collaboration and specifically how the project groups dealt with diverse ideas and perspectives and negotiated meaning. In sum, these studies had to make sense of the diverse data through the lens of intricate theoretical concepts to answer an explorative question. In these studies, it was practically not possible to make use of standardized procedures and tools for data gathering and data analyses. In both studies the researcher was faced with the problem of assuring the quality in data gathering and analyses processes. Moreover, it was difficult for the researcher to check and assess the maintained quality herself. Hence, an audit procedure seemed apparent as a way to evaluate the quality, and an audit trail seemed a useful tool to explicate all the decisions and according substantiations during the research process.

In the following sections, we will discuss the procedure as we further developed it.

\section{Guide for the Auditee: How to Produce an Audit-Trail?}

The audit trail is the material object for evaluation. As already mentioned, the audit trail is a documentation of the process of data gathering and data analysis. The researcher who conducts the study to be evaluated, the auditee, is responsible for creating an audit trail in such a way that it is complete and understandable for the auditor to evaluate. Several components are required to speak of a complete audit trail. In Table II, these components have been summarized. 
Table II. Audit-trail components

\begin{tabular}{ll}
\hline Materials & Description \\
\hline 1. Start document & This document encompasses the prob- \\
& lem, the conceptual framework or \\
& theoretical perspective, the (planned) \\
& methods and expected results, along \\
& with a reflection on the researcher \\
& position in the study. \\
& Thesis, journal article, conference \\
& paper, report. \\
& Raw data and field notes. Raw \\
2. Final document & materials often have to be made \\
& accessible to the auditor (e.g., taped \\
3. Raw data & conversations into written records, raw \\
& data with indexes or coding books). \\
& Processed data and memos. Raw \\
& data will often be processed before \\
& the analyses (coded records, summa- \\
& ries, annotated records and journals, \\
statistical results, etc.). \\
This document covers a systematic \\
report on the data gathering and \\
data analysis, in terms of the actions \\
undertaken and the associated results. \\
\hline 5. Process document &
\end{tabular}

To become familiar with the study, the audit trail should first of all contain a start document that reports on the state of affairs at the moment the study started. It includes the problem and research questions, the conceptual framework, the theoretical perspectives, and the methodological approach and design of the study. The start document also contains the expected results and a reflection of the researcher on his or her position in the study. The start document helps the auditor to understand the theoretical and methodological perspective claimed by the researcher, and to assess the study from these perspectives.

Second, the audit trail includes a final document. This document (thesis, journal article or conference paper) is actually the point of departure for conducting the audit. The final document shows the results, conclusions and interpretations claimed by the researcher on the basis of the conducted study. The auditor has to evaluate if these results and conclusions are in line with the (often invisible) processes of data gathering and analysis, and 


\section{AUDITING QUALITY}

if these processes are acceptable according to general standards. It must be clear that the auditor does not assess the quality of the report itself; since we reason that is commonly done by the reviewers or editors of a journal, or the supervisors in case of a thesis.

Third, the audit trail should contain all the raw as well as processed data that are object for the analyses. The raw data forms the empirical basis of the study, in which all interpretations should be grounded. Raw data might be accompanied by field notes, which are annotations of the researcher during the process of gathering data. Most of the times, these are methodological notes such as records of remarkable incidents or possible influences from the research context. Often data is being processed, that is, grouped into factors or dimensions, before the actual analysis. For example, taped interviews will be typed out and/or summarized, observations will be clustered based on time or themes, open-questions answers will be sorted and clustered. This whole process of memoing processed data and field notes supports the auditor to assess the link between raw data and analyses.

The final, but also key document in the audit trail is the process document that fully reflects the process of both data gathering and data analysis, in terms of the substantiated decisions taken by the researcher in those processes, along with the derived results. The focus is on the decisions actually taken, as the audit procedure is about the quality of the research and not about the quality of one's process to learn to do research. It is this document that has to make the research process visible and comprehensible, and has to demonstrate its acceptability. Within both the phases of collection and the analysis of the data, we distinguish activities that are planned from activities that are actually realized. We argue that it is relevant for the auditee to both prospectively as well as retrospectively document the data gathering and data analysis. This enables the researcher to read back and reflect on where certain activities might have turned out different than planned. Also, this makes aware that planned quality is not a guarantee for realized quality, as reality often turns out more complex. Subsequently, this means that the auditee describes decisions during both the planning and designing of data gathering instruments and analytic steps, as well as decisions taken during the actual data gathering and analysis, with the associated rationales. Besides the substantiated description of the decisions during planned and realized actions, there is a second type of component we claim to be important for the process document, namely quality notes. These notes reflect how the researcher attempted to maintain as well as check the quality of the research process in terms of possible bias that might have occurred in data gathering and data analysis. What has been done by the researcher during the design of data gathering and analysis purposefully to maintain quality, and how did 
the researcher check the quality of the actual gathering and analysis processes during or afterwards? Such notes in the audit trail allow the auditor to follow up whether the quality maintenance and assurance have led to satisfying outcomes during the research process. For example, maintaining quality of data gathering might include creating a safe environment for the interviewees or acquiring a prolonged field experience, whereas assuring the quality of data gathering refers to, for example, member checks or a check on steering questions of the interviewer. Maintaining the quality of data analysis includes an orientation on possible (statistical) analyses or a thick description, whereas assuring the quality refers to regular activities such as determining (interrater) reliability and various forms of validity. If quality maintenance and quality assurance have not been (fully) reported by the researcher, the auditor may perform quality checks him or herself, with the precondition that it must be possible for the auditor to conduct this. If not, the auditor only can write in the final evaluation report that the quality of specific parts of the research process was not evaluated in terms of the specific quality criteria and remains to be questionable.

In sum, the process document encompasses a description of the decisions during planned and during realized data gathering actions, and a description of the decisions during planned and during realized data analyses actions. The types of considerations that can be part of the substantiations of all these decisions are theoretical and methodological considerations, or considerations regarding quality maintenance or quality assurance. We strongly suggest this comprehensive way of reporting the research process in the audit trail, since it allows not only the auditor to evaluate the quality systematically, but also reveals where lack of quality resulted from. Moreover, reporting the process pro- and retrospectively, makes transparent to the researcher which aspects should be seriously considered to assure quality.

Besides making a complete audit trail, it is important to make the trail understandable for the auditor. This requires that the auditee creates a clear structure and writes perhaps more explicitly than would be necessary for one's own understanding. A suggestion for the auditee is to create an audit-trail index to inform the auditor which components are included, in what order, and what function they had in the research process. The trail may be organized in different ways, for example in chronological order of researcher's actions, or by research questions.

\section{Guide for the Auditor: How to Determine the Quality?}

As mentioned earlier, the audit procedure starts with an orientation to the audit and an orientation to the study. Following, the auditor looks into the audit trail and determines whether he or she thinks it is understandable and complete in such a way that the trail allows an assessment of the 
data gathering and data analysis. If so, the contract for the audit can be settled.

The actual assessment in stage 5 starts with verifying all linkages between research design, data gathering, data analysis, results, interpretations and conclusions. This verification is done in a systematic way, following the sequence of the trail, the research questions or main conclusions, or the chronological order of the steps of data gathering and data analysis. Of all methodological actions and decisions from research design up to conclusions, the auditor determines whether the criteria of visibility, comprehensibility, and acceptability have been met. Although the assessment can follow the chronological order of the research process, the conclusions and interpretations of the results as reported in the final document are the starting points. That is, the main underlying question for the auditor is whether these results and conclusions are grounded in the process of data gathering and data analysis in a way that the auditee made linkages that are visible (visibility), substantiated (comprehensibility), and logically and scientifically acceptable (acceptability).

This leaves open the interpretation of acceptability. Acceptability means that the substantiation of a research decision follows the argumentative logic of the field, and connects to what is considered as known and not known in the research domain. Hence, it becomes directly relevant for an auditor to see what field and accordingly, which standards, norms and values are being claimed by the researcher. This means that the auditor takes into account what research domain, and what theoretical and methodological position are being claimed by the researcher. These claims can be found in the final document and, more extensively, in the start document of the audit trail, and in the quality notes of the auditee about the kind of activities that were undertaken to maintain and determine the quality of gathering and analyzing the data.

The auditor presents his of her findings to the auditee. Following, the auditee assesses the accuracy of the auditor claims and whether the assessment reports what has been agreed. Discrepancies in understanding the audit trail and in the assessment of the study can be clarified by auditor and auditee. After this renegotiation the auditor writes the auditor report, including three major components:

- a summary of the specific audit procedure;

- the assessment of the quality of the study with respect to visibility, comprehensibility and acceptability, and

- an assessment scheme.

The summary of the audit procedure includes a description of the procedure with the activities and agreements that were specified for the particular case. Each of the stages of the audit procedure is described, along 
SANNE AKKERMAN ET AL.

\begin{tabular}{|c|c|c|c|c|}
\hline \multirow{2}{*}{\multicolumn{2}{|c|}{\begin{tabular}{|l} 
Audit-trail \\
components
\end{tabular}}} & \multicolumn{3}{|c|}{ Quality } \\
\hline & & \multirow[t]{2}{*}{ Visibility } & \multirow[t]{2}{*}{ Comprehensibility } & \multirow[t]{2}{*}{ Acceptability } \\
\hline Data & Planned & & & \\
\hline gathering & Realized & & & \\
\hline Data & Planned & & & \\
\hline analysis & Realized & & & \\
\hline
\end{tabular}

Figure 1. Basic structure of the assessment scheme in the audit report.

with the agreements between auditee and auditor, the renegotiation and the results of the renegotiation. Besides a more descriptive text about the assessment, we propose to include an assessment scheme, as in Figure 1, in which the conclusions of the auditor are systematically summarized. Each of the cells of the matrix in Figure 1 refers to the auditor's assessment of the specific information from the audit trail on the three criteria for trustworthiness of the study.

\section{Conclusion and Discussion}

Based on the original work of Halpern (1983), an audit procedure has been redesigned. In this procedure an external evaluator assesses the quality of a research study with the help of standardized guidelines. As such, this procedure is a solution for determining the quality of complex research that mostly is of a qualitative nature. Three generic criteria are formulated to determine the quality of scientific research: visibility (are decisions explicated and communicated?), comprehensibility (are these decisions substantiated?), and acceptability (are the substantiated decisions acceptable according to the standards, values and norms in the particular research domain?). On the basis of the materials produced by the auditee (the audit trail), an auditor assesses the quality of the study in terms of these three generic criteria (the auditor report). Guidelines for both the auditee and auditor were described including the main components of an audit trail and the type of assessments of the auditor.

The additional value of this audit procedure is that the maintenance of the quality through the work of the auditee as well as the assessment of the quality by the auditor are systematized into a procedure for quality assurance which covers the research decisions and researcher's interpretations. The essential task for both auditee and auditor is to link the interpretations and conclusions in the final research document to the data, through all steps in-between. Compared to other procedures for quality assurance 
in which external evaluators are used (interrater reliability, peer review or critical friends), the audit procedure is more comprehensive. Activities in terms of interrater reliability mostly are part of only testing measurement instruments, whereas an audit procedure covers the research process from data gathering to the interpretation of the results of the analyses. Moreover, peer review procedures are mainly focused on the products which have to be assessed. Finally, as in the audit procedure, the use of critical friends might be focused on the whole research process also, but is generally less structured and systematized. So, our conclusion is that the audit procedure has an additional value as a procedure for quality assurance in social scientific research. Moreover, the experience in conducting the audit in the two studies mentioned was that the recordkeeping system in the form of the audit trail, triggered the researchers' awareness of maintaining quality during the various research decisions. We therefore consider the audit procedure also as a good way to improve the quality of a study, in addition to the assessment of the quality.

With respect to the evaluation of the audit procedure which has been developed, the most obvious set of criteria to be applied on tools and procedures refers to their usability or practicality (see, e.g., Driessen, 2003). This set includes the comprehensibility of the procedures (clear structure of the audit trail, explicit wording in both the audit trail, and auditor report), the applicability (time and energy should be in line with the additional value of the audit procedure for determining the quality of research), attractiveness, and a match between the comprehensibility of the audit procedure and the competencies and skills of both the auditee and auditor. These criteria are related to the accuracy of the assessment of the quality of a study: the more clear and definite the audit trail and the more complete the assessment of the auditor, the higher the accuracy of the assessment of the quality of the study (cf., Funder, 1995). In general, our conclusions about the audit procedures are positive following these criteria, albeit still some work has to be done. We realize that the applicability of the procedure developed could be an issue, especially if resources are limited, researchers work in isolation or financers of a specific research project emphasize the practicality of research outcomes. The latter means that a researcher would not be able to spend much time to an audit procedure as this postpones conclusions which are based on the research study. We think there is a balance in the time the auditee spends on the audit trail and the effort the auditor puts in auditing the research: structuring the audit trail costs a lot of time of the auditee, but saves much time of the auditor. Yet, auditees who do not spend much time in structuring the audit trail ask much time from the auditor. It is our experience that it is worthwhile to spend relatively much time on the audit trail. In addition to saving time of the auditor, this helps the auditee to maintain the quality of the study. If 
there is not enough time available for the auditee or auditor, an alternative procedure would be to perform an audit procedure based on a sample of the data. Then, it should be possible to generalize the auditor's conclusions about the quality of that particular part of the study to the quality of the research project as a whole. How to audit a sample is one of the aspects we still have to work out.

Another issue about the audit procedure is the capabilities of both the auditee and auditor. Practising the audit procedure is a complex activity in itself. Producing an audit trail not only takes a lot of time, it is also a difficult task. This task requires the ability to reflect on the research process and to report the decisions that have been made. In this, it is important that the auditee reports the research decisions taken on the road between research problem and conclusions. The auditor too should have particular capabilities. An auditor must be an expert in the research domain, has to be able to assess a study from a methodological perspective, and should have an independent opinion about the research. If these criteria are met, supervisors of the researcher could act as auditor as they probably have time and are willing to evaluate the quality of the research. However, it should be clear that auditing a research is something different than supervising (the research and learning process of) a researcher.

A third issue to consider is the ambiguity of the object that is assessed. In the procedure described above, both the audit trail and the final document are on paper. This means that these products, in addition to the content of the audit trail, lean on form and style of writing. This means that there is a danger that the quality of the information provided for the auditor is dependent on the writing capabilities of the auditee. Ideally, we would say that the auditor should be capable to assess a research study whether the audit trail is well formulated or not. But, our experience is that this is not strictly the case in the audit practice. The way an audit trail and the final document have been written (with respect to form and style) does reflect on the content of the auditor report, and therefore on its quality assessment.

Finally, it is relevant to reflect on the generalizability of this audit procedure. We think this procedure is especially interesting in the case of complex research, which means a researcher does not have other standard strategies to assess the quality of the research and relies on many interpretations. We suppose the audit procedure (though in a more simple way) can also be used in less complex research. However, the additional value could be less, as there are already other, more simple procedures for quality assurance. The audit procedure could then be used as an overall procedure to evaluate whether the specific quality procedures used are suitable to cover the quality assurance of the whole process of data gathering and analysis. Another aspect of generalizability is whether the three main criteria 


\section{AUDITING QUALITY}

of visibility, comprehensibility and acceptability are shared as the criteria for the quality of scientific research. It could be that in some paradigms or research domains, other criteria are considered more important, like for example, voicing the target group (as in feminist research), or the practicality of the results of the research. In those cases, the audit procedure as developed in this study would be less suitable. In short, we believe the audit procedure to be most valuable in case a researcher aims to bring forward a study involving more complex research processes as rigorous.

\section{Notes}

1. The literature search was conducted in three databases: Education Resources Information Center (ERIC), PsycINFO, and Sociological Abstracts. The search, limited to articles published between January 1981 and November 2005, was performed by using the key words procedure and quality in a methodological context. Words used related to quality were objectivity, validity, reliability, accuracy, confirmability, dependability, trustworthiness, truth, and verification. Words used related to procedure were method, standard, guideline and criteria. Having selected the audit procedure, successive searches focused on audit, audit trail, reflexive journal, peer debriefing, and peer review. A total of 745 articles were found, of which 106 related to the audit trail as a procedure to determine the quality of scientific research: 292 in ERIC, 226 in PsycINFO and 227 in Sociological Abstracts. The researchers then screened the articles, first by title and then by abstract, to obtain articles relevant to the research problem. After screening, a total of some 50 articles were reviewed in full. Additional references were obtained from key articles - Guba (1981), Halpern (1983), Lincoln and Guba (1985), Schwandt and Halpern (1988) - and relevant authors' and publishers' websites. Besides, experts in the field were consulted.

2. We found these references in 52 documents involving 27 journal articles, 24 dissertations, and one book.

\section{References}

Akkerman, S. (2006). Strangers in Dialogue. Academic Collaboration across Organizational Boundaries. Unpublished doctoral dissertation, Utrecht, The Netherlands: Utrecht University.

Akkerman, S., Admiraal, W., Simons, R. J., \& Niessen, T. (in press). Considering diversity: Multivoicedness in international academic collaboration. Culture and Psychology.

Driessen, C. M. M. (2003). Analyzing Textbook Tasks and the Professional Development of Foreign Language Teachers. Utrecht: Utrecht University.

Funke, J. (1991). Solving complex problems: Exploration and control of complex systems. In: R. Sternberg and P. Frensch (eds.), Complex Problem Solving - Principles and Mechanisms. Hillsdale: Lawrence Erlbaum Associates, pp. 185-222.

Funder, D. C. (1995). On the accuracy of personality judgment: A realistic approach. Psychological Review 102:652-670.

Guba, E. G. (1981). Criteria for assessing the trustworthiness of naturalistic inquiries. Educational Communication and Technology Journal 29:75-91.

Halpern, E. S. (1983). Auditing Naturalistic Inquiries: The Development and Application of a Model. Unpublished doctoral dissertation, Indiana University.

Lincoln, Y. S. \& Guba, E. G. (1985). Naturalistic Inquiry. Newbury Park: Sage. 
Middleton, H. (2002). Complex problem solving in a workplace setting. International Journal of Educational Research 37(1):67-84.

Miller, D. L. (1997). One Strategy for Assessing the Trustworthiness of Qualitative Research: Operationalizing the External Audit. Paper Presented at the Annual Meeting of the American Educational Research Association, Chicago, IL.

Rodgers, B. L. \& Cowles, K. V. (1993). The qualitative research audit trail: a complex collection of documentation. Research in Nursing and Health 16:219-226.

Sale, J. E. M. \& Brazil, K. (2004). A strategy to identify critical appraisal criteria for primary mixed-method studies. Quality and Quantity 38:351-361.

Schwandt, T. A. \& Halpern, E. S. (1988). Linking Auditing and Metaevaluation: Enhancing Quality in Applied Research. Thousand Oaks, CA: Sage Publications Inc. 